\title{
Laser Defocusing Effects on Laser Ablation Inductively Coupled Plasma-Atomic Emission Spectrometry: Different Ablation Interactions between the Laser and Low-Alloy Steel, Fe Pellets, and a Pond Sediment Pellet
}

\author{
Masaki OHATA and Naoki FuruTA ${ }^{\dagger}$ \\ Department of Applied Chemistry, Faculty of Science and Engineering, Chuo University, \\ Kasuga, Bunkyo-ku, Tokyo 112-8551, Japan
}

\begin{abstract}
The ablation interaction between a laser and solid samples, which affects the analytical performance for laser ablation inductively coupled plasma atomic emission spectrometry (LA-ICP-AES), was studied. The emission intensities of elements observed by LA-ICP-AES (LA-ICP-AES element signal intensities) for different solid samples were measured under different laser defocusing conditions with a fixed laser output energy. It was found that the optimum laser defocusing conditions were dependent on the different solid samples with different sample characteristics, and also on the different elements with different elemental characteristics in each solid sample. A low-alloy steel, pellets containing different Fe concentrations ( $0-100 \%$ Fe pellet), and a pond sediment pellet were used as different solid samples. The variations of the LA-ICP-AES Fe signal intensities observed under different laser defocus conditions were completely different between the low-alloy steel and the pond sediment pellet. The changes in the LA-ICP-AES Fe signal intensities for 90 and $100 \%$ Fe pellets were similar to that of the low-alloy steel. However, pellets with lower Fe concentrations (less than 70\%) showed different trends and the defocusing behavior became closer to that of the pond sediment pellet. The LA-ICP-AES signal intensities of other elements were also evaluated, and were compared for different solid samples and different defocusing behavior. It was observed that the changes in the LA-ICP-AES signal intensities of almost all elements in the pond sediment pellet showed a similar trend to those of $\mathrm{Fe}$ for different laser defocus positions; that is, the elemental fractionation for these elements in the pond sediment pellet seemed to be relatively small. On the contrary, it was found that the LA-ICP-AES Si, Ti, and $\mathrm{Zr}$ signal intensities for low-alloy steel showed different trends compared to those of other elements, including Fe, under different defocusing conditions; that is, the elemental fractionation observed for the low-alloy steel was larger than that of the pond sediment pellet. From these results, different ablation interactions between the laser and the different solid samples were considered, and attributed to the sample characteristics, such as the matrix, hardness, and conductivity. Elemental fractionation was attempted to be explained by using elemental characteristics, such as the melting point and ionization energy of the elements.
\end{abstract}

(Received October 17, 2003; Accepted January 19, 2004)

\section{Introduction}

Recently, the use of laser ablation (LA) as a sampleintroduction system for inductively coupled plasma atomic emission spectrometry (LA-ICP-AES) $)^{1-5}$ and mass spectrometry (LA-ICP-MS) ${ }^{6-22}$ has been increasing for the determination of trace elements in solid samples. Although the technique is able to easily achieve qualitative and isotopic analysis, problems are always encountered when quantitative analysis is carried out. The main problem is that LA-ICP-AES/MS always needs a standard reference material (SRM) that has the same, or similar, matrix component compared to the target samples for quantitative analysis. The main reason is attributed to the different ablation interactions between the laser and the solid samples with different characteristics. In order to clarify the disadvantage, the ablation interactions between the laser and solid samples, ${ }^{5,18,21}$ ablated particle size distribution, ${ }^{6}$ deposited

$\doteqdot$ To whom correspondence should be addressed. particles on a transport tube, ${ }^{18,19}$ etc. have been investigated. Although these investigations were carried out to improve the analytical performance for LA-ICP-AES/MS, the situation which SRMs are required for calibration still remains at this moment.

The purpose of this study was to understand the ablation interactions between a laser and solid samples with different characteristics. In our previous work, ${ }^{5}$ the $\mathrm{Fe}$ emission signal intensities from a laser-induced plasma (LIP) and LA-ICP-AES for both a low-alloy steel and a pellet of pond sediment (pond sediment pellet) were observed under different laser defocus conditions. The largest LA-ICP-AES Fe signal intensity was observed under a laser on-focus condition for the low-alloy steel. On the other hand, defocused laser conditions gave larger LA-ICP-AES Fe signal intensities for the pond sediment pellet. The sample characteristics between the low-alloy steel and the pond sediment pellet used in a previous study were completely different; therefore, it was considered that an investigation of samples having intermediate characteristics between these two samples is necessary to further understand the ablation 
Table 1 Laser ablation system and ICP-AES operating conditions

\begin{tabular}{|c|c|}
\hline Laser ablation system & LSX-100 (Cetac) \\
\hline Laser & Nd:YAG \\
\hline Laser mode & Q-switched \\
\hline Wavelength & $266 \mathrm{~nm}$ \\
\hline Pulse duration & $8 \mathrm{~ns}$ \\
\hline Output energy & $2.1 \pm 0.1 \mathrm{~mJ}$ \\
\hline Frequency & $20 \mathrm{~Hz}$ \\
\hline Ablation time & $30 \mathrm{~s}$ \\
\hline Ablation mode & Raster \\
\hline ICP-AES & Flame Compact E (Spectro) \\
\hline Incident power & $1.2 \mathrm{~kW}$ \\
\hline Plasma gas flow rate & $16.01 \mathrm{~min}^{-1}$ \\
\hline Auxiliary gas flow rate & $1.261 \mathrm{~min}^{-1}$ \\
\hline Carrier gas flow rate & $0.771 \mathrm{~min}^{-1}$ \\
\hline Analysis mode & Time resolved analysis \\
\hline Integration time per point & $100 \mathrm{~ms}$ \\
\hline Measurement time & $60 \mathrm{~s}$ \\
\hline
\end{tabular}

interactions. In this study, we prepared pellets with various $\mathrm{Fe}$ concentrations $(0,10,30,50,70,90$, and 100\%). Three different types of solid samples, that is, low-alloy steel, Fe pellets, and a pond sediment pellet, were used and LA-ICP-AES $\mathrm{Fe}$ and other element signal intensities were observed under different laser defocus conditions to further understand the ablation interaction for improving the analytical performance of LA-ICP-AES/MS.

\section{Experimental}

Table 1 indicates the operating conditions of the instruments used in this study. The laser-ablation instrument used was a LSX-100 laser-ablation system (CETAC, Omaha, USA), employing UV $266 \mathrm{~nm}$ Nd:YAG laser. The laser output power $(2.1 \pm 0.1 \mathrm{~mJ})$ and laser shot frequency $(20 \mathrm{~Hz})$ were fixed during all experiments, but the laser focus positions were varied from 0 to $\pm 7 \mathrm{~mm}$ with respect to the surface of the solid samples. The crater diameter was increased with laser defocusing $(30,100,160$, and $220 \mathrm{~mm}$ under $0, \pm 1, \pm 2$, and \pm 3 $\mathrm{mm}$ laser defocus conditions, respectively, for the low-alloy steel). A laser pulse duration of $8 \mathrm{~ns}$ was also fixed; therefore, the power densities for the corresponding laser focus conditions changed $\left(37,3.3,1.3\right.$, and $\left.0.7 \mathrm{GW} \mathrm{cm}^{-2}\right){ }^{5}$ Because the laser ablation was conducted by a raster mode, the laser power densities could be fixed during laser ablation for each laser defocus condition.

A low-alloy steel standard (JSS 1008, Japanese Iron and Steel CRMs, The Iron and Steel of Japan, Tokyo, Japan) and a pond sediment pellet prepared from SRM NIES No. 2 pond sediment (National Institute for Environmental Studies, Tsukuba, Japan) were used to evaluate the LA-ICP-AES element signal intensities. To prepare the pond sediment pellet, a sample of pond sediment weighing $0.2 \mathrm{~g}$ was pelletized for $30 \mathrm{~min}$ at 30 $\mathrm{MPa}$. The diameter of the pellet was $10 \mathrm{~mm}$ and about $2 \mathrm{~mm}$ thick. Moreover, pellets containing different $\mathrm{Fe}$ concentrations of $0,10,30,50,70,90$, and $100 \%$ of $\mathrm{Fe}$ (Fe pellets) were also prepared by pressing using the same procedures as that for the pond sediment pellet. High-purity Fe powder (Johnson Matthey Materials Technology, UK) and $\mathrm{KBr}$ powder (Cica-Reagent,
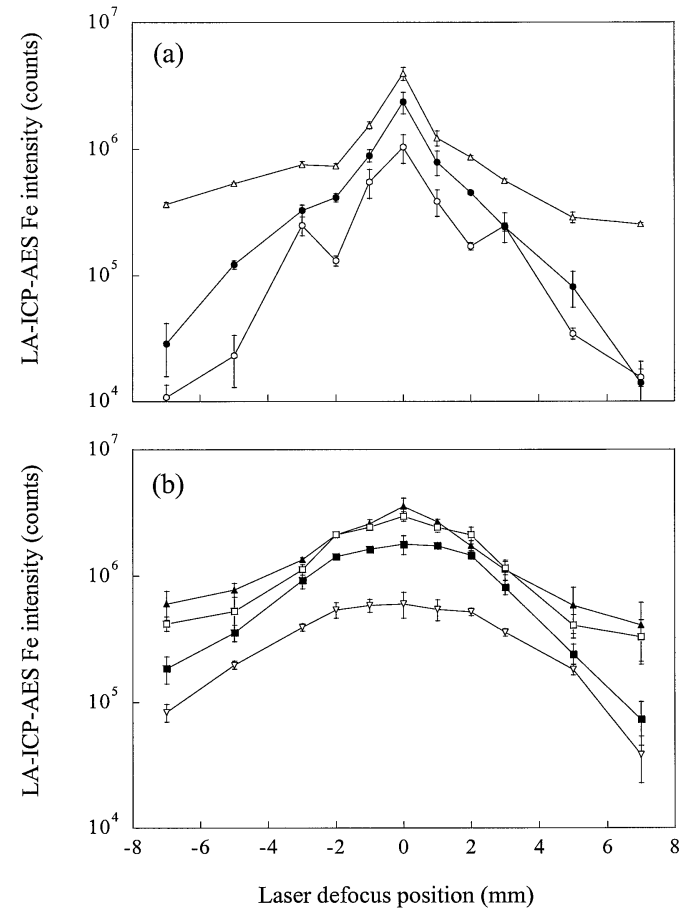

Fig. 1 LA-ICP-AES Fe signals vs. laser defocus positions: (a) 0 , low-alloy steel; •, $100 \% \mathrm{Fe} ; \triangle, 90 \% \mathrm{Fe}$. (b) $\Delta, 70 \% \mathrm{Fe} ; \square, 50 \%$ $\mathrm{Fe} ; \mathbf{\square}, 30 \% \mathrm{Fe} ; \nabla, 10 \% \mathrm{Fe}$ pellets.

Kanto Chemical Co. Inc., Tokyo, Japan) as a binder were mixed and ground using an agate mortar, and were then pelletized.

LA-ICP-AES element signal intensities were measured using Spectro Flame Compact E ICP-AES (Spectro. Co. Ltd., Kleve, Germany). The laser-ablation cell was connected to the ICP by a $3 \mathrm{~m}$ Tygon tube (1/8 inch i.d.). An ablation time of $30 \mathrm{~s}$ was fixed, i.e. 600 laser pulses $(20 \mathrm{~Hz})$ per measurement. A measurement of the LA-ICP-AES element signal intensities was conducted with a time-resolved analysis mode of the ICP-AES for $60 \mathrm{~s}$. The ICP-AES has two polychrometers and one monochromator; a maximum of 41 elements can be measured simultaneously. Depending on the concentrations of the elements in each solid sample, the number of elements observed was limited and different. The Fe I $373.5 \mathrm{~nm}$ atom line was selected by the monochromator when the Fe II $259.9 \mathrm{~nm}$ ion emission intensity is strong.

\section{Results and Discussion}

Evaluation of the LA-ICP-AES Fe signal intensities for lowalloy steel, $\mathrm{Fe}$ pellets, and a pond sediment pellet

Figure 1 shows the LA-ICP-AES $\mathrm{Fe}$ signal intensities observed for pellets with different $\mathrm{Fe}$ concentrations as a function of the laser defocus positions as well as those for the low-alloy steel. It can be seen from Fig. 1 that the largest LAICP-AES Fe signal intensity could be observed at the on-focus position for the low-alloy steel and Fe pellets. Then, the LAICP-AES Fe signal intensities decreased with increasing the laser defocusing. However, it could be seen that different variations of the LA-ICP-AES Fe signal intensities were found for pellets with different Fe concentrations. The pellet with 90 and $100 \%$ Fe concentration showed a similar trend to that of the low-alloy steel (Fig. 1(a)). However, the differences in the LAICP-AES Fe signal intensities between the on-focus and defocus 


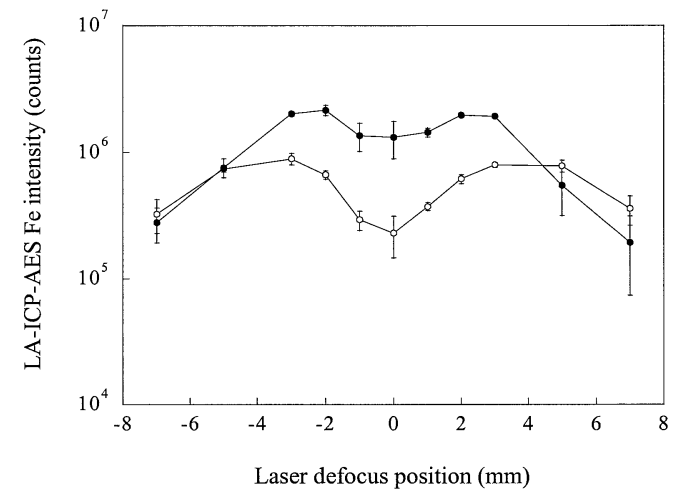

Fig. 2 LA-ICP-AES Fe signals vs. laser defocus positions: $0,0 \%$ Fe pellet (Fe II $259.9 \mathrm{~nm}$ ion line); •, pond sediment pellets (Fe I $373.5 \mathrm{~nm}$ atom line)

conditions (from 0 to $\pm 2 \mathrm{~mm}$ laser defocus condition) became smaller for Fe pellets with an Fe concentration less than $70 \%$ (Fig. 1(b)). From these results, it can be considered that the different LA-ICP-AES Fe signal intensities are likely to be dependent on the sample conductivity. Although the conductivity of the low-alloy steel is not available in the literature, it can be estimated to be about $80 \mathrm{~W} \mathrm{~m}^{-1} \mathrm{~K}^{-1}$ at $273 \mathrm{~K}$ from the data of pure iron $\left(76 \mathrm{~W} \mathrm{~m}^{-1} \mathrm{~K}^{-1}\right.$ at $273 \mathrm{~K}^{-1}$ and $80.2 \mathrm{~W}$ $\mathrm{m}^{-1} \mathrm{~K}^{-1}$ at $\left.300 \mathrm{~K}\right){ }^{23}$ The laser power density, which is strongly related to the photon energy from the laser and the heat energy from the LIP, and the shock wave from the laser can be considered to be important interaction factors between the laser and a solid sample. The ablation interaction between the laser and samples with high conductivity (low-alloy steel, 90 and $100 \% \mathrm{Fe}$ pellets) is mainly dependent on the laser power density. On the other hand, it is considered that the ablation interaction is dependent on not only the laser power density, but also on the laser irradiated area when the sample conductivity becomes low (pellets with an Fe concentration less than 70\%). The increasing of the laser-irradiated area compensated for the decrease of the laser power density. Since ablation mechanisms due to a shock wave require less energy; this fact implies that the shock wave from the laser is more predominant than the photon energy from the laser and the heat energy from the LIP.

Figure 2 shows the LA-ICP-AES Fe signal intensities obtained for both a $0 \% \mathrm{Fe}$ pellet (Fe concomitant in $\mathrm{KBr}$ pellet, the conductivity of $\mathrm{KBr}$ was reported as $5 \mathrm{~W} \mathrm{~m}^{-1} \mathrm{~K}^{-1}$ at $\left.273 \mathrm{~K}\right)^{23}$ and the pond sediment pellet (the conductivity of the pond sediment is not available in the literature, but is estimated to be similar to that of the sand data of $0.33 \mathrm{~W} \mathrm{~m}^{-1} \mathrm{~K}^{-1}$ at $293 \mathrm{~K}$ ). ${ }^{23} \mathrm{~A}$ measurement of the LA-ICP-AES Fe signal intensity for the $0 \%$ Fe pellet was conducted using the Fe II $259.9 \mathrm{~nm}$ ion line of ICP-AES, because the Fe concentration in the $100 \% \mathrm{KBr}$ pellet was too low to measure the $\mathrm{Fe}$ emission intensities using the $\mathrm{Fe}$ I $373.5 \mathrm{~nm}$ atom line. As can be seen from Fig. 2, the LA-ICPAES Fe signal intensities were enhanced by laser defocusing from 0 to $\pm 3 \mathrm{~mm}$, and then decreased. The laser defocusing behaviors for both the $0 \% \mathrm{Fe}$ pellet and the pond sediment pellet were similar. However, the LA-ICP-AES Fe signal intensities did not show exactly the same trend, because the matrix component between these two samples was completely different. Because the defocused laser leads to a decrease in the laser power density, the ablation interaction can be considered to be dependent on the shock wave mainly when the conductivity of the sample is low $(0 \%$ Fe pellet and the pond sediment). Since the interaction between the laser and these

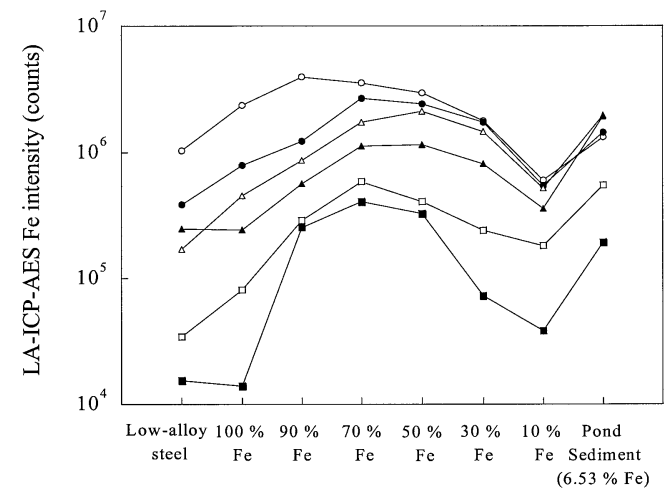

Fig. 3 LA-ICP-AES Fe signal intensity for the low-alloy steel, 10 $100 \% \mathrm{Fe}$ pellets and the pond sediment pellet for different laser defocus conditions: $\bigcirc$, on-focus; $\bullet, 1 \mathrm{~mm}$ defocus; $\triangle, 2 \mathrm{~mm}$ defocus; $\Delta, 3 \mathrm{~mm}$ defocus; $\square, 5 \mathrm{~mm}$ defocus; $\mathbf{\square}, 7 \mathrm{~mm}$ defocus.

two samples is substantially a shock wave, the signal enhancement could be observed by increasing the laser defocusing, which resulted in an increase in the laser irradiated area. Moreover, it could be concluded that the matrix difference also influences the ablation interaction between the laser and the solid samples.

In Fig. 3, the LA-ICP-AES Fe signal intensities are plotted for different samples under different laser defocus conditions. From this figure, a higher LA-ICP-AES Fe signal intensity for the $100 \%$ Fe pellet was observed compared to that of the lowalloy steel. The difference in the hardness between the lowalloy steel and the $100 \%$ Fe pellet can be considered to be the reason for this intensity difference. It was also found that the LA-ICP-AES Fe signal intensities were enhanced when the sample conductivity became low for each laser defocus condition. The intensity of the $100 \%$ Fe pellet was smaller compared to that of the 90 and $70 \%$ Fe pellets. Since the hardness of pressed pellets can be considered to be similar for these Fe pellets, it can be evaluated that the ablated amounts increased when the sample conductivity became low. On the other hand, the LA-ICP-AES Fe emission intensities for the $10 \% \mathrm{Fe}$ pellet were smaller than those of the pond sediment pellet $(6.53 \% \mathrm{Fe})$. The difference in the signal intensity is not only dependent on the conductivity, but also on the matrix difference between the $90 \% \mathrm{KBr}$ in the $10 \%$ Fe pellet and the $45.8 \% \mathrm{SiO}_{2}$ in the pond sediment pellet.

Evaluation of the LA-ICP-AES element signal intensities for low-alloy steel, Fe pellets, and a pond sediment pellet

The changes in the LA-ICP-AES signal intensities for elements other than Fe were evaluated in order to discuss the elemental fractionation for different laser defocusing and for solid samples with different characteristics. Figures 4(a) and (b) show the relative intensities of the LA-ICP-AES element signal intensities for the low-alloy steel and the pond sediment pellet, respectively. The relative intensities were calculated for each element based on the LA-ICP-AES element signal intensities observed at the on-focus position. Only the relative intensities for each element observed at positive defocus positions $(0-7$ $\mathrm{mm}$ ) are shown, because the resultant trends observed at minus defocus positions showed the same trend as the positive ones. From Fig. 4(a), it can be seen the differences in the relative intensities between $\mathrm{Fe}$ and the other elements at the $1 \mathrm{~mm}$ defocus position are small, except for $\mathrm{Si}$ and $\mathrm{Zr}$. When the laser defocus position was more than $2 \mathrm{~mm}$, the difference in the 

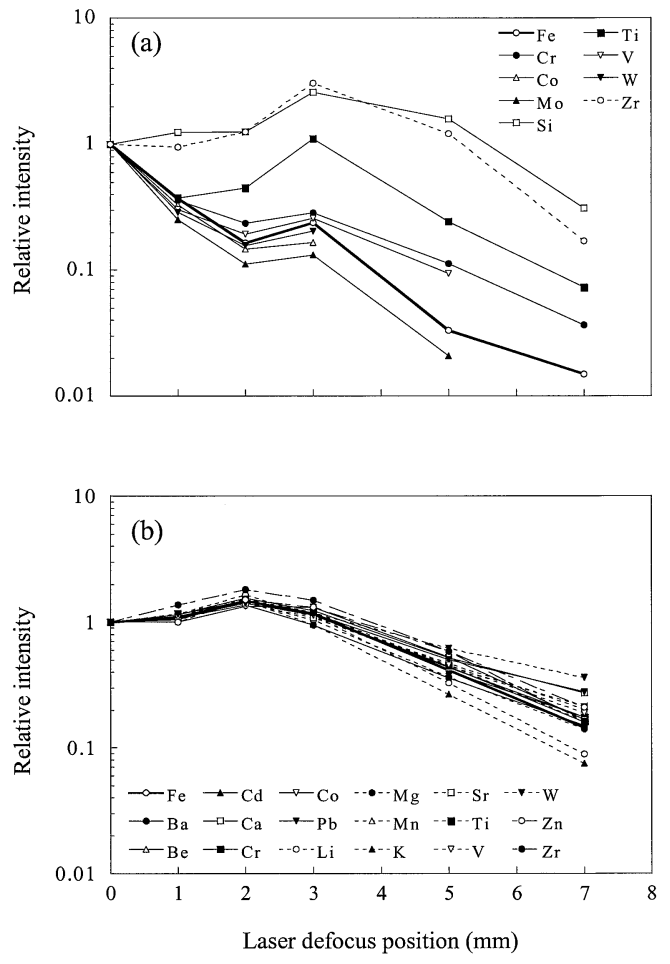

Fig. 4 Relative intensities of LA-ICP-AES element signals $v s$. laser defocus positions: (a) low-alloy steel and (b) pond sediment pellet.

relative intensities became slightly larger (particularly $\mathrm{Ti}$ ). However, most of the elements ( $\mathrm{Cr}, \mathrm{Co}, \mathrm{Mo}, \mathrm{V}$, and $\mathrm{W}$ ) showed a similar decreasing trend compared to that of $\mathrm{Fe}$. As mentioned in the previous section, because the interaction between the laser and the samples with high conductivity is mainly dependent on the laser power density, the signals for these elements decreased with increasing the laser defocusing. In addition, a similar laser defocusing trend means that the ablation behavior between $\mathrm{Fe}$ and these elements is similar; that is, the elemental fractionation for these elements can be evaluated as being small. On the other hand, the trends of the relative intensities for $\mathrm{Si}, \mathrm{Ti}$, and $\mathrm{Zr}$ showed large differences compared to those of $\mathrm{Fe}$ for all laser defocus conditions (except for $\mathrm{Ti}$ at the $1 \mathrm{~mm}$ laser defocus position). From these results, it can be concluded that the elemental fractionation for $\mathrm{Si}, \mathrm{Ti}$, and $\mathrm{Zr}$ is larger than that for other elements $(\mathrm{Cr}, \mathrm{Co}, \mathrm{Mo}, \mathrm{V}$, and $\mathrm{W})$. Since these signals for $\mathrm{Si}, \mathrm{Ti}$, and $\mathrm{Zr}$ were enhanced with increasing the laser defocusing, it can be considered that the ablation interaction is related to the laser-irradiated area. On the contrary, the variations in the relative intensities for different elements observed for the pond sediment pellet showed a similar trend as a function of the laser defocus positions (Fig. 4(b)).

The same evaluations were also conducted for all Fe pellets. Figures 5(a) - (c) show the relative intensities of the LA-ICPAES element signal intensities observed for the $90 \%, 50 \%$, and $0 \% \mathrm{Fe}$ pellets, respectively. The element signals are due to the concomitants in both Fe powder and $\mathrm{KBr}$. That is, the different mixing ratio for the preparation of the $0-100 \%$ Fe pellets leads to a different concentration of the elements; thus, the detectable elements were different for pellets with different $\mathrm{Fe}$ concentrations. From Fig. 5, it can be found that the variations of the relative intensity were different for pellets with different $\mathrm{Fe}$ concentrations. In Fig. 5(a), $\mathrm{Cr}$ and $\mathrm{Fe}$ show a similar decreasing trend as a function of the laser defocus positions;
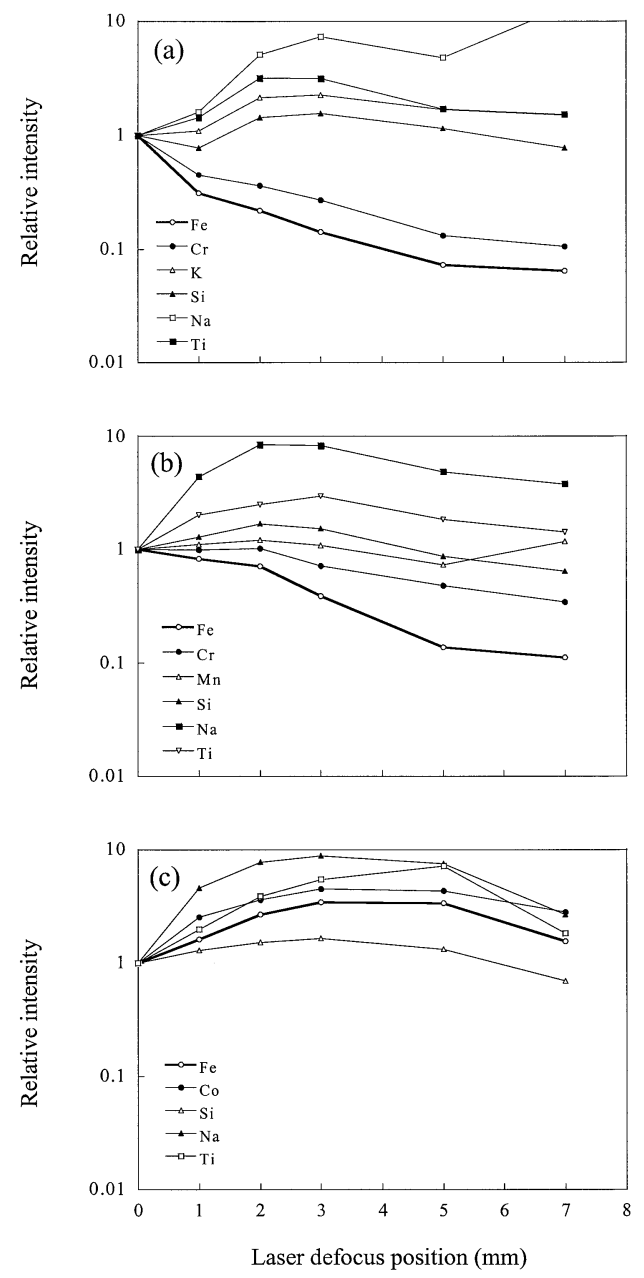

Fig. 5 Relative intensities of LA-ICP-AES element signals vs. laser defocus positions: (a) $90 \% \mathrm{Fe}$, (b) $50 \% \mathrm{Fe}$ and (c) $0 \% \mathrm{Fe}$ (concomitant in $\mathrm{KBr}$ ) pellets.

however, other elements (K, $\mathrm{Si}, \mathrm{Na}$, and $\mathrm{Ti}$ ) were enhanced and then decreased. Moreover, the laser defocusing effects were different for $\mathrm{K}, \mathrm{Si}, \mathrm{Na}$, and $\mathrm{Ti}$. On the other hand, all of the elements observed were enhanced and then decreased for the 0\% Fe pellet, as shown in Fig. 5(c). The degree of elemental fractionation seems to be smaller with decreasing an $\mathrm{Fe}$ concentration. The laser defocusing effects of the $50 \%$ Fe pellet (Fig. 5(b)) revealed an intermediate behavior between that of the $0 \%$ and $90 \% \mathrm{Fe}$ pellets. From these results, it can be concluded that the different elemental fractionations are dependent on the sample characteristics.

Evaluation of the ablation interactions between the laser and elements in low-alloy steel, Fe pellets, and a pond sediment pellet

When the sample conductivity is high, larger elemental fractionation seems to occur compared to that of a sample with low conductivity. Figures $6-8$ show the fractionation index of elements in the low-alloy steel, the $50 \%$ Fe pellet, and the pond sediment pellet, respectively. The fractionation index is the ratio value calculated as the relative intensity of LA-ICP-AES element divided by the relative intensity of LA-ICP-AES Fe at each laser defocus position. That is, Figs. $6-8$ were obtained based on the results shown in Fig. 4(a), Fig. 5(b), and Fig. 4(b), respectively. If the fractionation indexes for the element are 

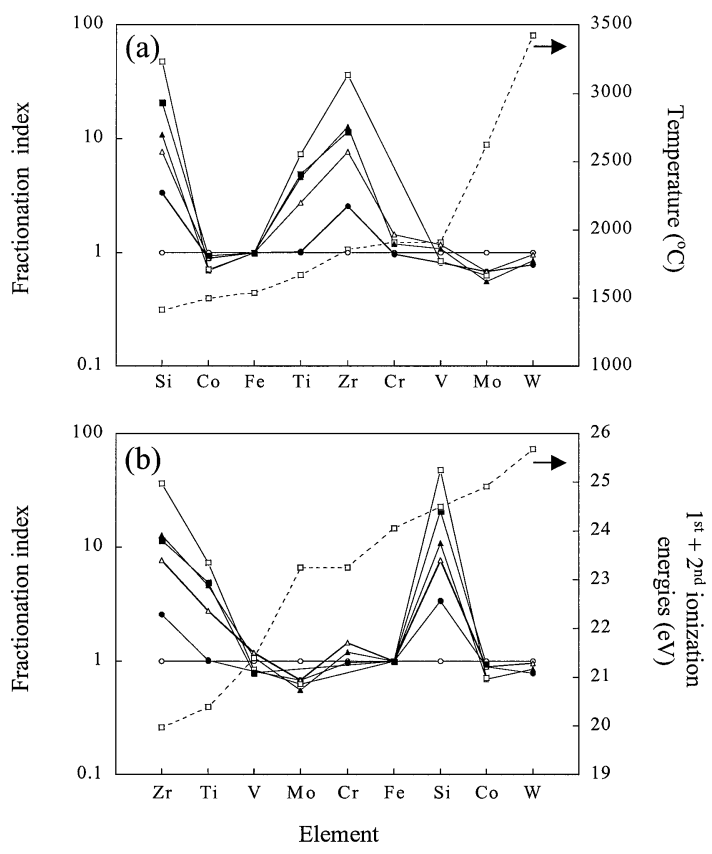

Fig. 6 Fractionation index of different elements in the low-alloy steel in the ascending order of (a) melting point and (b) 1st plus 2nd ionization energies: $O$, on-focus; $\bullet, 1 \mathrm{~mm}$ defocus; $\triangle, 2 \mathrm{~mm}$ defocus; $\mathbf{\Delta}, 3 \mathrm{~mm}$ defocus; $\square, 5 \mathrm{~mm}$ defocus; $\mathbf{\square}, 7 \mathrm{~mm}$ defocus.

close to 1 under different laser defocusing conditions, it can be evaluated that the ablation behavior of the element is similar to that of Fe. That is, the elemental fractionation can be evaluated as being small. The fractionation indexes are plotted in ascending order of (a) the melting point and (b) addition of the first and the second ionization energies of the elements.

It can be seen from Fig. 6(a) that the fractionation index of $\mathrm{Si}$, which has a lower melting point compared to other elements, was large with an increase in the laser defocusing. It seems that the elements which have lower melting point are easily ablated; thus, an increase of the laser-irradiated area compensates for a decrease of the laser power density. However, $\mathrm{Ti}$ and $\mathrm{Zr}$ also showed large fraction indexes. This contradiction can be explained by the fact that addition of the first and the second ionization energies of $\mathrm{Ti}$ and $\mathrm{Zr}$ is low as shown in Fig. 6(b). As can be seen in Fig. 6(b), it also seems that the elements which have a lower ionization energy are easily ablated; thus, an increase of the laser-irradiated area compensates for a decrease of the laser power density. A similar ablation mechanism is proposed in Ref. 22.

It can be seen from Fig. 7 that the results observed for the $50 \% \mathrm{Fe}$ pellet were similar to those of the low-alloy steel, as shown in Fig. 6. The elements that have a lower melting point and a lower ionization energy showed larger fractionation indexes.

As can be seen from Fig. 8, the fractionation index observed for the pond sediment pellet was close to 1 for almost all elements. It can be considered that the difference in the fractionation index between the low-alloy steel and the pond sediment pellet is dependent on the different ablation interactions, which is attributed to the different sample characteristics. For the pond sediment pellet, the primary ablation interactions can be presumed to be a shock wave during laser irradiation, because the hardness is low compared to that of the low-alloy steel. In this case, the elemental fractionation for different elements is small, because the ablation interaction
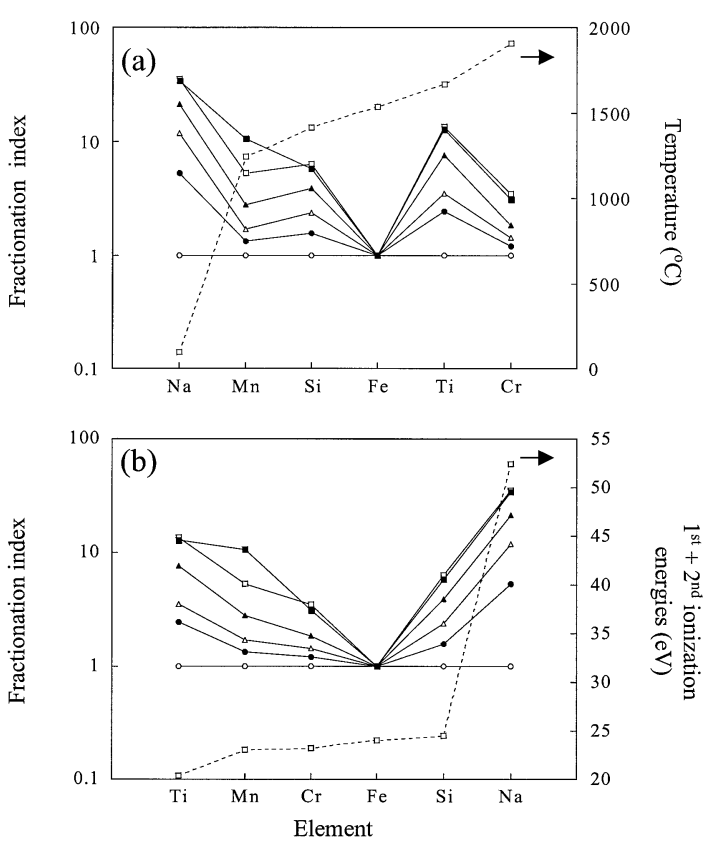

Fig. 7 Fractionation index of different elements in $50 \%$ Fe pellet in the ascending order of (a) melting point and (b) 1st plus 2nd ionization energies of elements: $\bigcirc$, on-focus; $\bullet, 1 \mathrm{~mm}$ defocus; $\triangle, 2$ $\mathrm{mm}$ defocus; $\mathbf{\Delta}, 3 \mathrm{~mm}$ defocus; $\square, 5 \mathrm{~mm}$ defocus; $\mathbf{}, 7 \mathrm{~mm}$ defocus.
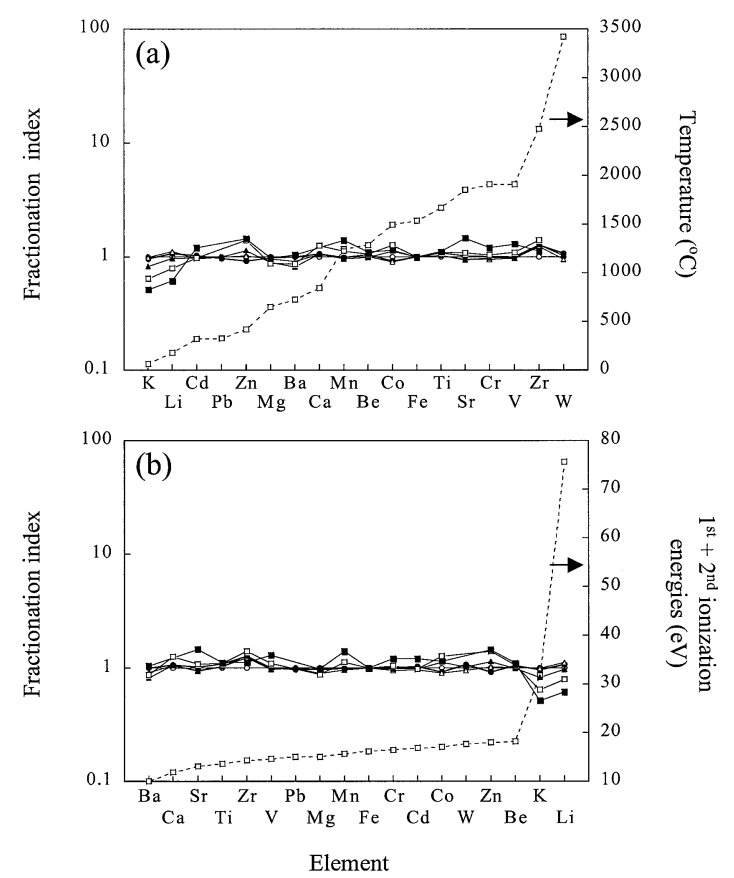

Fig. 8 Fractionation index of different elements in the pond sediment pellet in the ascending order of (a) melting point and (b) $1 \mathrm{st}$ plus 2nd ionization energies of elements: 0 , on-focus; $\bullet, 1 \mathrm{~mm}$ defocus; $\triangle, 2 \mathrm{~mm}$ defocus; $\mathbf{\Lambda}, 3 \mathrm{~mm}$ defocus; $\square, 5 \mathrm{~mm}$ defocus; $7 \mathrm{~mm}$ defocus.

between the laser and the pellet is not dependent on the elemental parameters, such as the melting point and ionization energy. On the contrary, the low-alloy steel and Fe pellets with a higher $\mathrm{Fe}$ concentration showed a larger elemental fractionation compared to that of the pond sediment pellet. Because the conductivity of these samples is higher than that of 
the pond sediment pellet, the ablation interaction can be presumed to be related to the laser power density. From these results, it is concluded that the elemental characteristics, such as the melting point and addition of the first and second ionization energies, seem to be important factors for the elemental fractionation for samples with high conductivity.

\section{Conclusions}

The ablation interaction between the laser and the solid samples was investigated for low-alloy steel, $0-100 \% \mathrm{Fe}$ pellets, and a pond sediment pellet by using LA-ICP-AES in this study. From the obtained results, it can be concluded that the ablation interactions between the laser and the different samples depend on the sample characteristics, such as the matrix, hardness, and conductivity. In the case of samples with high conductivity, the ablation interaction is mainly dependent on the laser power density, which strongly affects the photon energy from the laser and the heat energy from the LIP; moreover, the elemental characteristics, such as the melting point and ionization energy, also influence the elemental fractionation. That is, elementally representative ablation can not be obtained easily; therefore, the corresponding SRMs are always necessary for the quantitative analysis of these samples. In the case of samples with low hardness and low conductivity, like the pond sediment pellet, the ablation interaction is mainly due to the shock wave from the laser; therefore, the smallest elemental fractionation, that is, the elementally representative ablation, can be obtained much more easily compared to that for samples with high conductivity. Consequently, quantitative analysis can be achieved using only one powdered SRM without precise matrix matching.

\section{References}

1. M. Hemmerlin, D. Somas, C. Dubuisson, F. Loisy, E. Poussel, and J. M. Mermet, Fresenius J. Anal. Chem., 2000, 368,31 .

2. M. Hemmerlin, J. M. Mermet, M. Bertucci, and P.
Zydowicz, Spectrochim. Acta Part B, 1997, 51, 421.

3. V. Kanicky, V. Otruba, and J. M. Mermet, Spectrochim. Acta Part B, 2000, 55, 575.

4. P. Musil, V. Otruba, V. Kanicky, and J. M. Mermet, Spectrochim. Acta Part B, 2000, 55, 1747.

5. M. Ohata, Y. Iwasaki, N. Furuta, and I. B. Brenner, Spectrochim. Acta, Part B, 2002, 57, 1713.

6. S. H. Jeong, O. V. Borisov, J. H. Yoo, X. L. Mao, and R. E. Russo, Anal. Chem., 1999, 71, 5123.

7. M. M. Heino, S. Rauch, G. M. Morrison, and O. F. X. Donard, Anal. Chim. Acta, 2001, 436, 233.

8. T. Hirata, J. Anal. At. Spectrom., 2002, 17, 204.

9. P. H. Chi, F. H. Ko, C. T. Hsu, H. L. Chen, C. K. Yang, Y. C. Sun, and M. H. Yang, J. Anal. At. Spectrom., 2002, 17, 358.

10. G. Tao, Y. Fujikawa, M. Mitsui, and R. Yamada, J. Anal. At. Spectrom., 2002, 17, 560.

11. N. H. Bings, J. Anal. At. Spectrom., 2002, 17, 759.

12. J. Feldmann, A. Kindness, and P. Ek, J. Anal. At. Spectrom., 2002, 17, 813.

13. A. M. Leach and G. M. Hieftje, J. Anal. At. Spectrom., 2002, 17, 852 .

14. P. R. D. Mason and W. J. Kraan, J. Anal. At. Spectrom., 2002, 17, 858 .

15. T. Prohaska, C. Latkoczy, G. Schultheis, M. T. Nicola, and G. Stingeder, J. Anal. At. Spectrom., 2002, 17, 887.

16. P. Marshall, O. Heudi, S. Bains, H. N. Freeman, F. A. Shakra, and K. Reardon, Analyst, 2002, 127, 459.

17. D. Gunther, Anal. Bioanal. Chem., 2002, 372, 31.

18. R. E. Russo, X. Mao, H. Liu, J. Gonzalez, and S. S. Mao, Talanta, 2002, 57, 425.

19. J. Koch, I. Feldmann, N. Jakubowski, and K. Niemax, Spectrochim. Acta Part B, 2002, 57, 975.

20. M. Ohata, H. Yasuda, Y. Namai, and N. Furuta, Anal. Sci., 2002, 18, 1105.

21. R. E. Russo, X. Mao, and S. S. Mao, Anal. Chem., 2002, 74, 71A.

22. Z. X. Chen, J. Anal. At. Spectrom., 1999, 14, 1823.

23. D. R. Lide, "Handbook of Chemistry and Physics", 79th ed., 1998, CRC Press LLC. 